\title{
Does attention move or spread during mental curve tracing?
}

\author{
David Crundall, Richard Dewhurst, and Geoffrey Underwood \\ University of Nottingham, Nottingham, England
}

\begin{abstract}
There are two theories that attempt to explain how attention is deployed when lines are traced. Initially, it was believed that a covert zoom lens moved along the line. Recent evidence has, however, suggested that attention spreads along the line, rather than moving along it, perhaps as part of an effortful object-parsing process. Three experiments tested the spreading and moving accounts of line tracing. Participants were presented with two intertwined lines and were required to trace one to find the correct target. On half the trials, a masked change occurred, most often near the top of the target line, that reversed the required response. If attention spreads along the line, the participants should have been able to notice the change whenever it occurred during the tracing process. However, the participants found it harder to spot the change if it occurred late in the tracing process. This suggests that resources were less frequently available to detect changes on portions of the line that had already been traced when the change occurred. The results argue against a spreading trace of attention that encompasses the whole line.
\end{abstract}

One of the most enduring questions in attention research has been how covert attention shifts between areas of interest. Recent evidence has suggested that orientation of attention is independent of the distance to be moved (e.g., Hazlett \& Woldorff, 2004), although greater distances may require more planning prior to a shift in attention. There are circumstances, however, in which an analogue or serial movement of covert attention is necessary. If participants are asked to judge whether two targets appear on the same convoluted line, their response times (RTs) increase with a corresponding increase in the separation between the two targets along that line, despite a constant Euclidean distance between them. This finding was initially reported by Jolicœur, Ullman, and Mackay (1986). They presented participants with two convoluted lines and asked them to judge whether a target at the point of fixation was on the same line as a second target in extrafoveal vision. Although the second target was always the same distance from fixation, the increased RTs for targets separated by a greater line length led to the suggestion that the line must be traced by a serial attentional operator.

In a series of subsequent experiments (e.g., Jolicœur \& Ingleton, 1991; Jolicœur, Ullman, \& Mackay, 1986, 1991; McCormick \& Jolicœur, 1991), it was found that the linetracing effect occurred with extremely short stimulus durations (ruling out any confounds due to eye movements); that the proximity of the distractor curve to the target curve affected RTs, with closer distractors resulting in slower tracing times; and that line tracing could also be slowed by the increasing curvature of a curve, but not by the overall size of the pattern. McCormick and Jolicœur (1994) examined a number of potential models to explain line tracing. Their preferred model posited a variable width spotlight, or zoom lens, that moved along the line, changing diameter constantly with the proximity of distractor lines. This model accounted for the majority of the effects noted in their experiments and was an improvement over the other serial operator models that they considered.

Recently, however, researchers have questioned the underlying mechanism involved in the line-tracing process. Scholte, Spekreijse, and Roelfsema (2001) suggested that line tracing is not achieved via a moving spotlight but, instead, involves a gradual spread of attention that eventually covers the whole line. They distinguished this from the zoom lens model of McCormick and Jolicœur (1994), which they assumed does not leave attentional resources attached to the portions of a curve that it has already passed over. In fact, in a later article, they even noted that were the zoom lens to leave an attentional trace in its wake, "the two models become identical" (Houtkamp, Spekreijse, \& Roelfsema, 2003, p. 1142). It is this crucial difference between the models that was examined by Scholte et al. and, subsequently, by Houtkamp et al. The following section will briefly describe these studies. Before proceeding, however, it should be noted that McCormick and Jolicœur (1994) made no claims regarding the portions of the line that the zoom lens operator had passed over. In fact, they suggested that line tracing might have an important role to play in figure-ground separation (McCormick \& Jolicœur, 1992), which implicitly suggests that some form of residual trace might be left behind. Although this reduces the clarity of the distinction suggested by Scholte et al., it does not invalidate it, since any trace left by McCormick and Jolicœur's (1994)

D. Crundall, david.crundall@nottingham.ac.uk 
zoom lens operator would have to have fewer resources devoted to it than at the current focus of attention. Otherwise, the spotlight hypothesis would indeed be identical to the spreading attention hypothesis. For the remainder of this article, we will adhere to Scholte et al.'s conception of the two models, with the spread hypothesis leaving a complete trace indistinguishable from the leading edge of the spread in regard to allocated resources, whereas the zoom lens hypothesis leaves either no trace or at least a trace with reduced resources, as compared with attention at the focus of the spotlight.

Scholte et al. (2001) were explicit in specifying the role that they believed line tracing could play in everyday tasks. They suggested that this process can be viewed as a secondary level of object parsing. The first level of object parsing, which they termed base grouping, is an almost immediate parsing of objects of varying complexity by shape-selective cells in the visual cortex. They recognized that some of these neurons may be quite high up in the processing hierarchy, in order to quickly parse common yet complex objects. When this process fails, usually because the object is so uncommon that there would be no advantage to having neurons specifically attuned to its presence, a second process of incremental grouping is required. Once attention has spread throughout the stimulus, the features are then bound together to provide the percept of that object (Roelfsema, Lamme, \& Spekreijse, 2000). They argued that this second process can also explain line tracing.

In support of a spreading trace of attention, Scholte et al. (2001) found that a secondary-task response to a colored segment of a target line was more accurate than a response to a colored segment on a distractor line. In this particular study, they used two symmetrical lines, one of which emerged from the fixation point and wound its way down the screen to connect with one of two potential target circles positioned at the bottom left and bottom right of the stimulus. The circle that was not connected by the target line to the fixation point was attached to the distractor line. Example stimuli can be seen in Figure 1. In addition, the arches of both the distractor and the target lines could appear in one of six different colors. The color of the arches was irrelevant to the primary task, which required participants to say whether the fixation point was connected to the bottom left or the bottom right circle. After this initial response, the participants were presented with a 300-msec color mask, before the lines reappeared without the colored segments. A spatial cue then prompted the participants to report the former color of one of the arches. They found that RTs to the primary task tended to slow with increased difficulty of the tracing task (i.e., the more often that the two lines crossed) and that color accuracy was higher for arches at all locations on the target line, as compared with the distractor line. On the basis of these findings, they rejected the hypotheses that the lines were traced in parallel or that attention jumped from one critical intersection to another (since this would have resulted in none of the colors' receiving attention). Instead, they explained the effects as incremental grouping via the spread of attention across the whole object.

Although there can be no question that attention was required to trace the target line in the Scholte et al. (2001) study, the distinction between a serial zoom lens or a spreading trace is less clear. Although they concluded in favor of the latter, one could equally well imagine a serial spotlight passing over the colored arches and storing this information in memory (especially if this information is relevant to a secondary task). This removes the need for attention to be present at all points on the line at the same time to explain the results.

A subsequent study by Houtkamp et al. (2003) provides much more compelling evidence for a spreading trace over a serial operator. They used the same figures as Scholte et al. (2001), although the colors appeared on the lines at varying stimulus onset asynchronies (SOAs) from
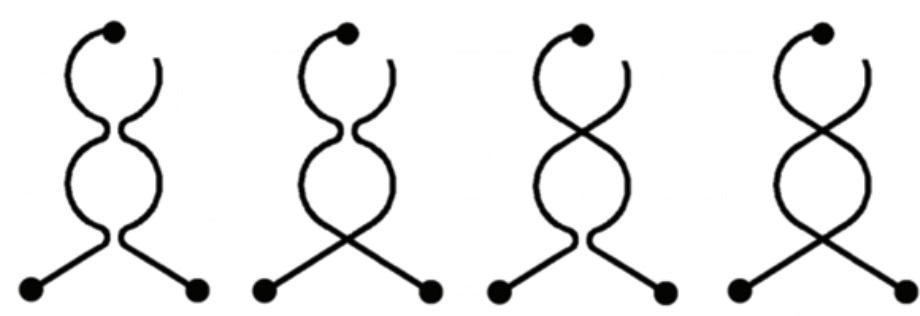

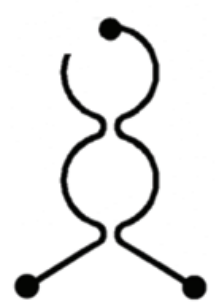

$\mathrm{OO}$

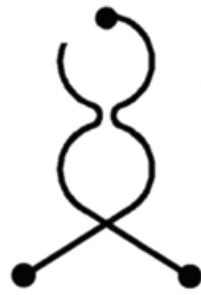

OX

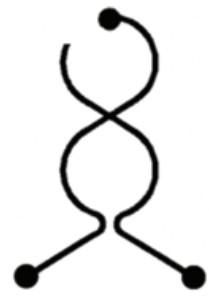

XO

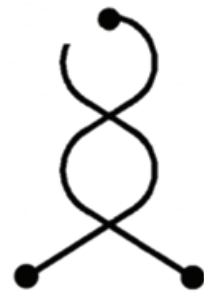

XX

Figure 1. The eight basic stimuli. 
the onset of the lines themselves. At short SOAs, both a serial zoom lens and a spread of attention should result in high accuracy for color segments near the top of the target line, as compared with color segments that appear at the bottom of the target line, because the line-tracing process will have only just started and both models predict that attention will be available near the top of the line. However, at relatively longer SOAs, the two models differ in their predictions. Although both models predict equal success at detecting color segments near the bottom of the target line at a long SOA, only the spreading attention hypothesis predicts that high accuracy will be maintained for colors at the top of the target line. The serial operator model predicts, instead, that accuracy for colors at the top of the target line will decrease as the SOA increases, since it is more likely that the spotlight will have already passed over the arch where the color appears, leaving no attentional resources to process it.

The results of Houtkamp et al.'s (2003) study revealed a pattern of secondary-task accuracy in which colors at the top of the target line were reported with a high degree of accuracy at all SOAs. Thus, they concluded in favor of the spread-of-attention model, rather than the serial operator model.

However, there are still several potential confounds that may have influenced the findings of Houtkamp et al. (2003). The use of a secondary task, for instance, may have a strategic effect upon the way that participants deploy their attention. A further problem with a secondary task is that the abrupt color onsets may disrupt the tracing process. In an attempt to remove this possibility, they used isoluminant color onsets, on the basis of evidence that suggested that color per se does not attract attention (e.g., Cole, Kentridge, \& Heywood, 2005; Theeuwes, 1995). There is, however, conflicting evidence that argues that isoluminant color onsets can still attract attention (e.g., Lu, 2006; Sumner, Adamjee, \& Mollon, 2002). In the absence of a baseline control condition (in which the stimuli should have been presented without color onsets), it is impossible to judge whether these abrupt onsets had any distracting effect. Furthermore, if the line-tracing process allows figure-ground separation (as has been suggested by McCormick \& Jolicœur [1992] and as also is compatible with Scholte et al.'s [2001] incremental grouping process), responses to the colors on the distractor line may be degraded due to the need to switch between figure and ground (Wong \& Weisstein, 1983). This could explain the results of Houtkamp et al. without necessarily requiring attention to be currently allocated to portions of the curve that have already been traced.

These potential confounds in the Houtkamp et al. (2003) study leave open the question regarding which of the two models of attention accounts for line tracing. In order to properly distinguish between the spreading attention model and the serial zoom lens model, we undertook the present experiments in which a single line-tracing task was used to avoid the potential confounds that may arise from a secondary task. Stimuli similar to those used by Houtkamp et al. were employed, with a probe appearing at either short or long SOAs after the tracing process had begun. The probe, which occurred on half of the trials, switched the required response by changing the target line into the distractor line. This was done by presenting a second stimulus identical to the first in all respects, except that the original target line was no longer connected to the fixation circle. Instead, the previous distractor line was connected to the fixation circle.

In an additional change to the methodology in Houtkamp et al. (2003), the onset of the second stimulus was masked by a global flicker. Many studies of change blindness have demonstrated that the inclusion of such a flicker swamps the local transients that are associated with abrupt onsets (Rensink, O'Regan, \& Clark, 1997; Simons, 1996), thereby removing the possibility that attention might be captured by the localized change in the stimulus.

It was predicted that if participants noticed the change to the stimulus after the flicker, they would realize that they were tracing the wrong line and would then begin the tracing process again, following the new target line. If, however, the participants did not perceive the change, they would continue the tracing process along the new distractor line and give the wrong answer. The important factor is the SOA at which the flicker and change occur. If the change occurs after a relatively short period of time, both the spreading trace and the serial zoom lens models predict that participants will spot the change and will switch to the new target line, resulting in increased RTs, although accuracy will be maintained. At long SOAs, however, the spreading trace theory predicts that accuracy at spotting the change will remain undiminished, whereas the zoom lens hypothesis predicts that as the focus of attention moves away from the critical area in which the change appears, participants will be less likely to notice the change and task accuracy will decline.

\section{EXPERIMENT 1}

\section{Method}

Participants. Sixteen participants were recruited to take part in the study (with a mean age of 20 years; 11 were female). All the participants reported normal or corrected-to-normal eyesight.

Stimuli and Apparatus. The stimuli were similar to those used by Houtkamp et al. (2003). A black circle was located at the top of the stimulus, coincident with the participants' point of fixation at the time of stimulus presentation, and two target circles were located $7.8^{\circ}$ below the fixation point and $2.6^{\circ}$ to either the left or the right of the central meridian. The fixation point was always connected to one of the target circles by a convoluted line, whereas a second distractor line was connected to the other target circle, but not to the fixation point. The target line crossed the path of the distractor line on three quarters of the stimuli. On one quarter of the stimuli, the lines crossed at $3^{\circ}$ below fixation, whereas one quarter of stimuli had paths that crossed at $6^{\circ}$ below fixation. The final quarter of trials crossed at both points. These four different types of trials are referred to as $o o, o x, x o$, and $x x$ trials, where the letters refer to the number of times the lines cross and in which location. For instance, the target line in an $o x$ trial crosses the distractor line only once, $6^{\circ}$ below the fixation point (i.e., the lower of the two potential locations where the lines may cross). In addition, the target line connecting the top circle to the target circle came from either the left side of the top circle or the right side, with equal probability. In total, there were eight basic stimuli (see Figure 1). For the main part of the experiment, a blue screen was inserted into each trial to provide a global flicker. 
The stimuli were presented on a P3 PC with a 21-in. FD Trinitron CRT monitor. A chinrest was used to maintain the distance between the screen and the participant, and a suitably angled mirror allowed the experimenter to ensure that the participant maintained fixation throughout each trial.

Design. There were two within-group factors that addressed the main hypothesis. The first was whether the target response changed or remained the same after a brief global flicker. Half of the trials involved a change of stimulus, where the original stimulus would be replaced by a similar stimulus, the only difference being that the target line would stem from the opposite side of the circle at the point of fixation. The effect of this change was to reverse the required answer. Where a target line emerging from the right of fixation might require a response indicating that the circle on the bottom left of the image was the target circle (as would occur with either an $o x$ or an $x o$ stimulus), after a change the correct response would also change to the target circle on the right. The second factor of importance to the main hypothesis was the SOA. In order to use SOAs that were scaled to individual performances, all the participants completed a baseline block prior to the main experimental blocks. These trials did not include a global flicker or a change to the stimulus and merely recorded RTs and the accuracy of the participants in identifying the correct target circle. The mean correct RT for each participant then had $200 \mathrm{msec}$ deducted from it (to remove some of the motor component of the response), and $10 \%$ and $60 \%$ of the remaining time was used for the individual short and long SOAs, respectively, for each participant (rounding to the nearest $10 \mathrm{msec}$ ).

The baseline block consisted of 96 trials made up from the eight basic stimuli. There were three experimental blocks of 128 trials each, with all of the eight stimuli equally represented; half of the stimuli were followed by a change, whereas the other half were not. Visual feedback ("correct" or "incorrect") was provided for the participants after each trial. During the experiment, the experimenter monitored the participants' eye movements throughout via a mirror. It was the intention that any participant with excessive eye movements would be removed from subsequent analysis.

Responses were made by the participants using the " 4 " and "6" keys on the numerical keypad of the computer keyboard. These keys also have left and right arrows on them, respectively. The participants were allowed to reposition the keyboard if they had a left-hand preference. Accuracy and RTs were recorded.

Procedure. The participants were seated $88 \mathrm{~cm}$ from the monitor, with head position stabilized in a chinrest. Instructions were presented on the screen and emphasized the need for accuracy and speed, as well as the need to keep the eyes focused at the point of fixation throughout the experiment. The instructions for the experimental blocks also told the participants about the global flicker and that, on some trials, the stimulus might change after the flicker, reversing the required response.

For the baseline block, each trial began with a fixation cross at the top of the screen that was available for $500 \mathrm{msec}$, followed by a stimulus for up to $2,000 \mathrm{msec}$, although this could be terminated if a response was made. If the participants did not respond within this time, they were presented with a message asking them to speed up. After visual feedback, the fixation cross returned for the next trial. After each participant had completed the baseline block, the short and long SOAs for that individual were calculated and were included in the subsequent experimental blocks. The sequence of events in an experimental trial can be viewed in Figure 2. The participants were given a brief practice of 10 trials (containing the flicker, randomly occurring at either short or long SOAs) before they started the three experimental blocks. At the end of each experimental block, the participant could take a break before resuming.

\section{Results}

The first part of the experiment required the participants to undertake a block of trials that did not contain the global flicker and did not contain any changes. This block of 96 trials, split evenly across the different stimulus types, was intended to provide baseline RTs on which to base the SOAs from stimulus onset to the global flicker used in the second part of the experiment. Both correct RTs and accuracy were collected. The mean RT on correct trials was $879 \mathrm{msec}$, and the average percentage number of trials correctly responded to was $82 \%$ across participants. The RT and accuracy scores for the baseline block were broken down into the four stimuli types (oo, ox, xo, and $x x$ ) and were compared in a $1 \times 4$ ANOVA. Repeated planned contrasts were employed to compare the levels of stimulus type in an order that we believed would reflect the increasing complexity of the tracing process (oo was compared with $o x$, $o x$ with $x o$, and $x o$ with $x x$ ). All the results are given with Cohen's $f$ as a measure of effect size.

Main effects of stimulus type were found for both RTs $\left[F(3,45)=30.0, M S_{\mathrm{e}}=6,631.6, p<.001\right.$, Cohen's $f=$ $1.4]$ and accuracy $\left[F(3,45)=12.1, M S_{\mathrm{e}}=195.3, p<.001\right.$,
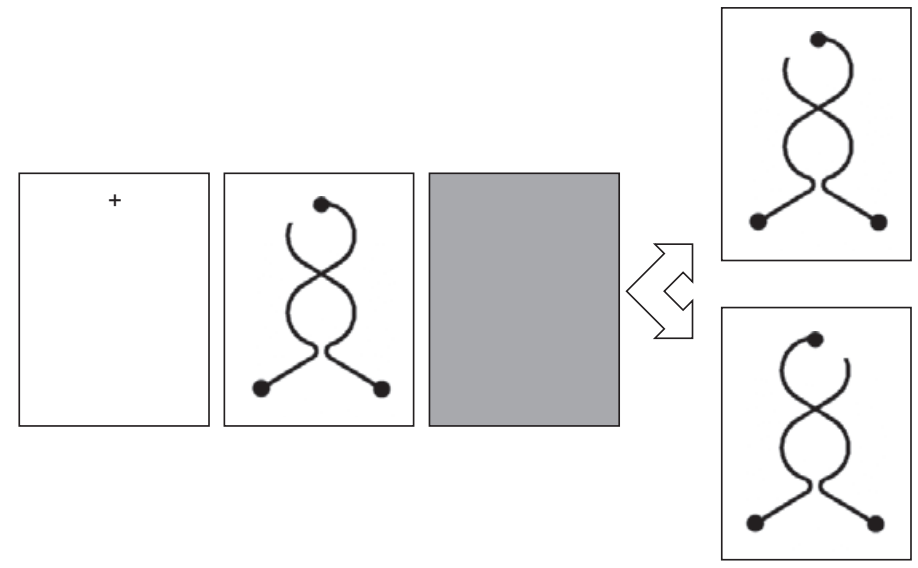
$f=0.9]$. The RTs followed the predicted direction, with oo trials producing the shortest RTs and $x x$ trials producing the longest RTs. The accuracy scores were not as straightforward. Although oo trials were the most accurate, there was an increase in accuracy for $x o$ trials, as compared with $o x$ trials (see Figure 3). This was possibly due to the dropoff in visual acuity across the retina that made the bottom intersection ( $6^{\circ}$ from fixation) harder to process than the top intersection ( $3^{\circ}$ from fixation), resulting in more errors with the more difficult crossover intersections. Although we cannot provide a conclusive explanation for this effect, it does mirror the error results that were found by Scholte et al. (2001) in their first experiment. Although the difference in tracing times according to the number of crossovers is not crucial to the main hypothesis of this experiment, it was comforting to note that the results were similar to previously published findings.

On the basis of the mean RTs in the baseline condition, short and long SOAs were calculated for each participant for the second part of the experiment with the global flicker. The average short SOA across participants was $71 \mathrm{msec}$, ranging from 30 to $100 \mathrm{msec}$. The average long SOA was $404 \mathrm{msec}$, ranging from 160 to $550 \mathrm{msec}$ for individual participants.

Accuracy and RTs were collected from 384 trials across three blocks. Trials were removed from the analyses if a response was made before the flicker. This amounted to $7.8 \%$ of all the responses. No participants needed to be removed

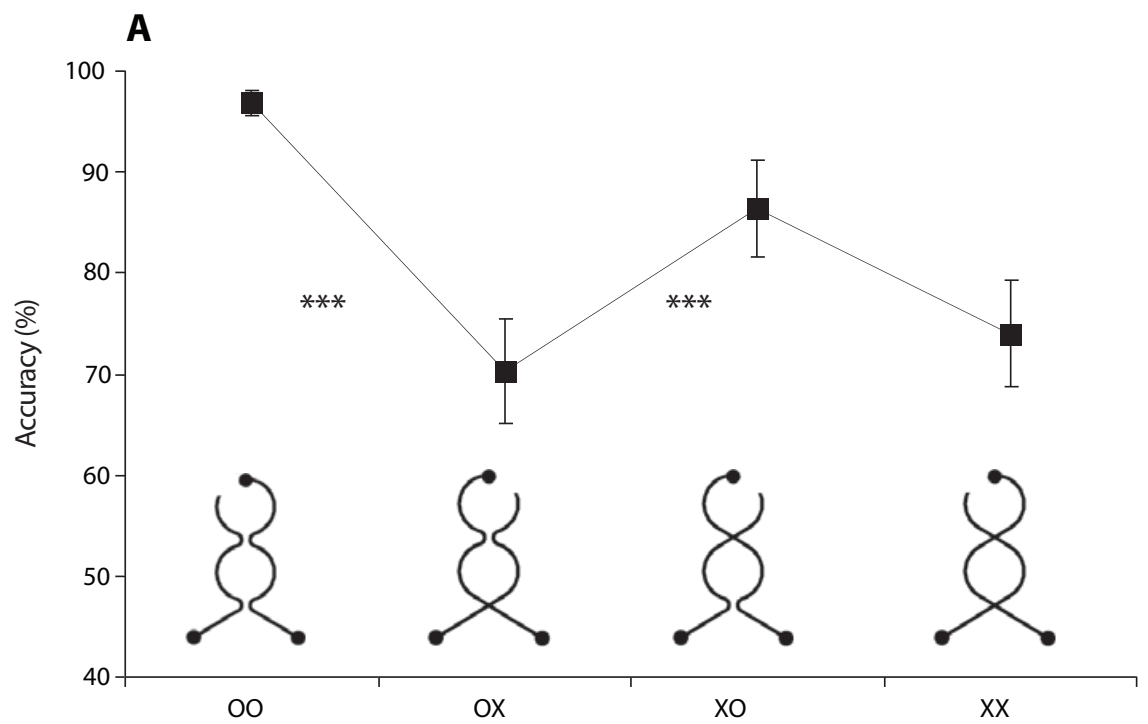

B

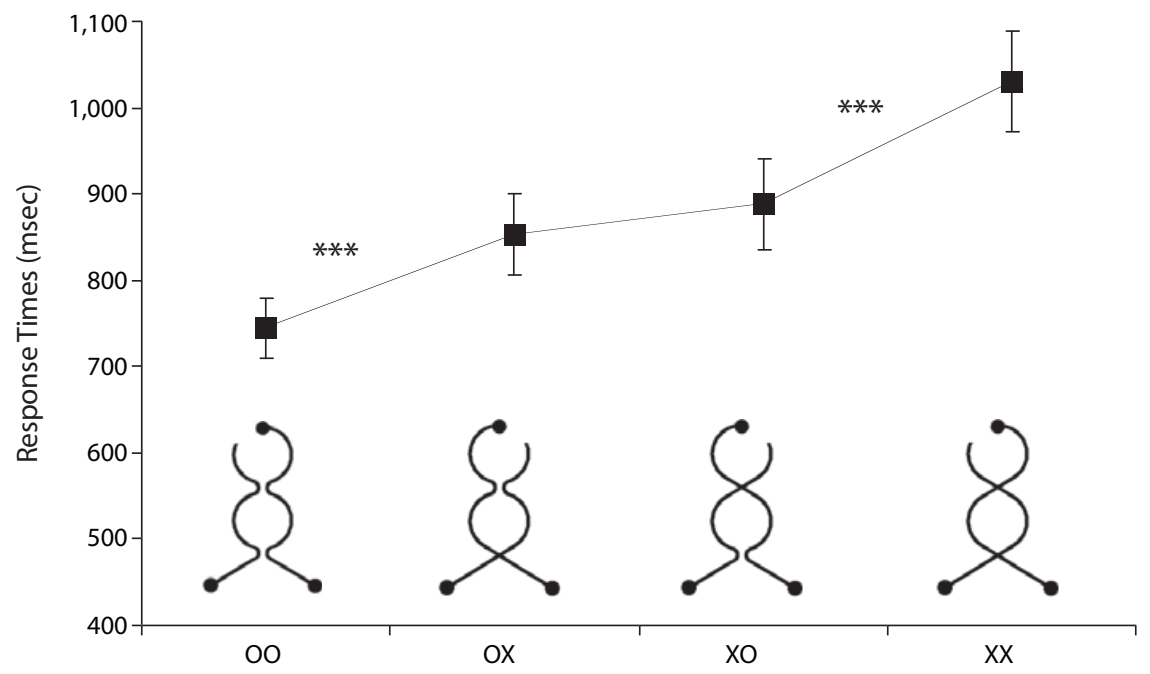

Figure 3. Mean percent correct (A) and response time (B) for the baseline block in Experiment 1. Standard error bars are added. ${ }^{* * *}$ Difference significant at $p<.001$, using repeated planned contrasts. 
for excessive eye movements. On average, eye movements were made by the participants during the experiment blocks on only $2.6 \%$ of all the trials (ranging from less than $1 \%$ to $4.5 \%$ across all participants). The accuracy scores were subjected to a $2 \times 2$ ANOVA, comparing the change/no-change conditions across the short and long SOAs. A main effect of change was noted $\left[F(1,15)=40.7, M S_{\mathrm{e}}=114, p<.001\right.$, $f=1.65]$. Since the nature of the change was to reverse the target response part way through the trial, it was expected that performance would drop on change trials (producing an average of $67.3 \%$ correct responses when the target response changed, as compared with $84.4 \%$ in the no-change trials). A main effect of SOA was also noted $\left[F(1,15)=8.9, M S_{\mathrm{e}}=\right.$ $76.7, p<.01, f=0.77$ ] with longer SOAs from stimulus onset to the global flicker coinciding with reduced accuracy, as compared with shorter SOAs $(72.5 \%$ and $79.1 \%$, respectively). More importantly, an interaction between the two factors was noted $\left[F(1,15)=27.8, M S_{\mathrm{e}}=38.1, p<.001\right.$, $f=1.36]$. This interaction (charted in Figure 4A) shows that the SOA between stimulus onset and the global flicker did not in itself affect accuracy, since there was little difference between the long and the short SOA conditions when the flicker was not accompanied by a change. However, the SOA was extremely important when the flicker was followed immediately by a reversal of the target response. Although all the change trials had a detrimental effect, regardless of SOA when compared with the no-change condition, the participants in the short SOA trials were still $75 \%$ accurate, on average. This suggests that on the greater majority of short SOA trials, the participants detected the change and reversed their response. Accuracy for the long SOA trials, however, fell dramatically to $60 \%$, suggesting that attention was less likely to be in a position to detect the change at long SOAs than at short SOAs.

Although the change detection hypothesis relies primarily upon the accuracy of responses, it was considered worthwhile analyzing the RTs for correct responses to assess whether a speed-accuracy trade-off could account for the decrease in accuracy in the long-SOA change condition. The mean RTs for correct answers were entered into a $2 \times 2$ within-group ANOVA, comparing the change and the nochange conditions across short and long SOAs. All RTs were recorded from the onset of the original stimulus. This analysis was first conducted on the data with $7.8 \%$ of the responses removed where the participant responded before the flicker. The analysis was then repeated with these trials left in, although the differences between the two patterns of results was negligible and did not affect the statistical results. The following data refer to the first of the two analyses.

Both main effects were found to be significant. RTs on changed trials were longer than those on trials without a change [969 and $813 \mathrm{msec}$, respectively; $F(1,15)=76.8$, $\left.M S_{\mathrm{e}}=5,024, p<.001, f=2.3\right]$, and short SOAs produced quicker RTs than did trials with long SOAs [775 and $1,007 \mathrm{msec} ; F(1,15)=66.8, M S_{\mathrm{e}}=12,871, p<.001$, $f=2.11]$. The interaction between the two factors was also significant $\left[F(1,15)=35.4, M S_{\mathrm{e}}=1,156, p<.001\right.$, $f=1.53]$ and can be viewed in Figure 4B. The interaction reflects the greater difference between the two levels of SOA in the change condition, as compared with the no-
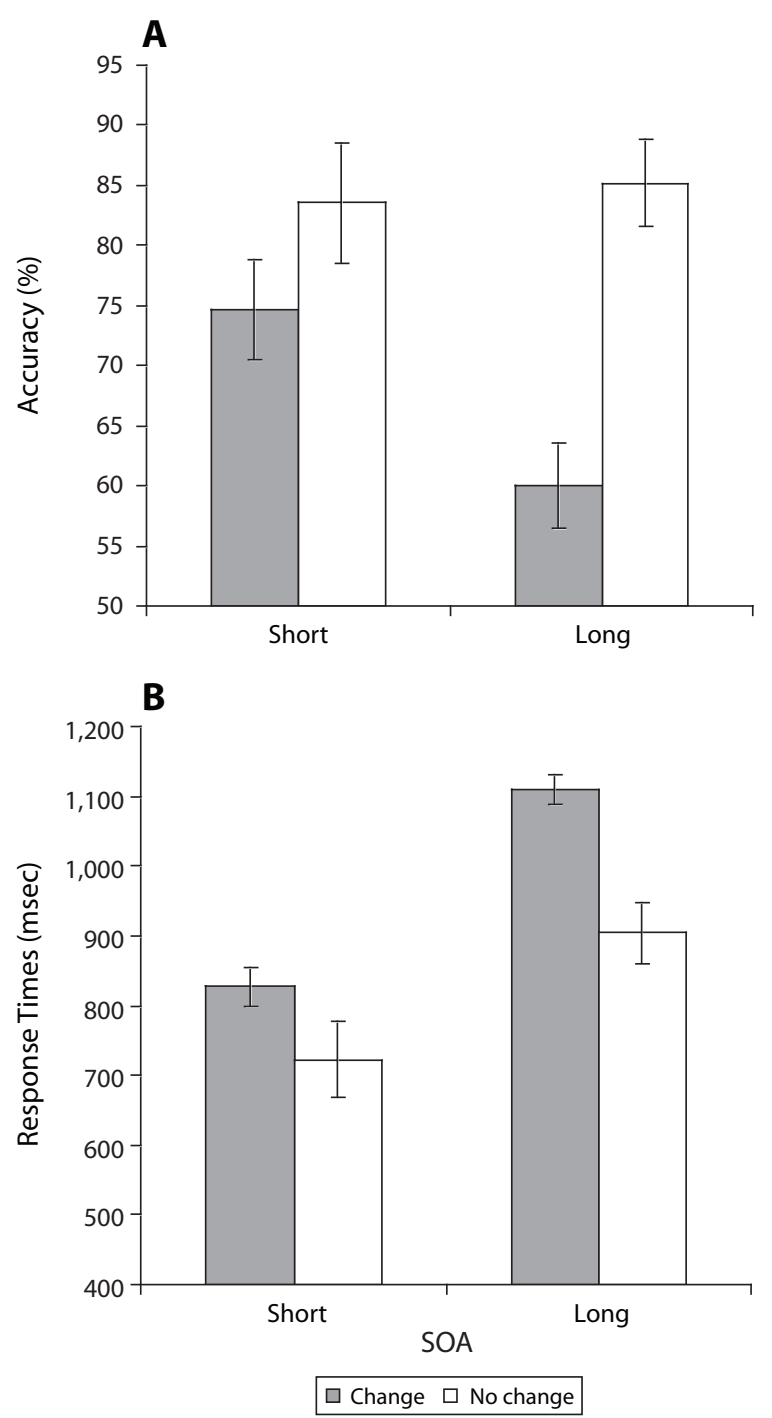

Figure 4. Accuracy of responses (A) and mean response times (B) to the correct target circle in Experiment 1. Note that in the change condition, the required response is reversed part way through the trial. Standard error bars are added.

change condition. Although a short SOA produces relatively short RTs, the onset of a change is more detrimental to RTs when it occurs at long SOAs. This suggests that the decreased accuracy for changed targets at long SOAs was not due to a speed-accuracy trade-off.

\section{Discussion}

The results have demonstrated that participants are more likely to notice a change at the beginning of a target line, when the tracing process has only just begun. If the change occurs at longer SOAs, however, when the tracing process may have progressed further along the line, participants are less likely to notice the change. It is generally considered that, under most conditions, attention to a stimulus in a visual scene or array is necessary to overcome change blind- 
ness (e.g., Rensink, 2000; Simons, 2000). Scholte et al. (2001) and Houtkamp et al. (2003) argued that attention is available at all portions of the line that have been traced and, therefore, that the change should have been detected regardless of SOA. However, the fact that change detection was poorer in the long-SOA conditions than in the shortSOA conditions fits with the serial zoom lens model, in which, according to Houtkamp et al., the zoom lens moves along the line without leaving any attention in its wake.

Despite the interaction's arguing against a simple spread of attentional resources, accuracy was still relatively high after changes at long SOAs (60\%). Does this mean that an attenuated trail of resources is left behind after the serial zoom lens has passed? Or does it simply reflect the fact that on a certain number of trials, the participants retrace the line from the top after the flicker occurs, just to make sure of their answer? It is possible that both of these processes could contribute to the relatively high accuracy, although the increased RTs in the condition indeed suggest that a subsequent line-tracing process may be undertaken on some trials, whereas on other trials (especially at long SOAs), the participants are successfully tricked into giving the wrong answer following a change. It is not necessary however to distinguish between these two post hoc explanations in order to conclude that these data do not fit with a strict spreading attention model.

It should also be noted that the choice of short and long SOAs, although systematic across participants, was arbitrary to a certain extent. It is possible that the long SOAs used in this experiment were not long enough. They were based on $60 \%$ of the baseline RT (after deducting $200 \mathrm{msec}$ for motor RT), although this was a subjective criterion that allowed us to consider only the relative accuracy rates between conditions and not the absolute values.

There is, however, a different potential problem with the choice of SOA levels that may have contributed to this result. The SOA levels were calculated per individual on the basis of their overall RTs to all the baseline stimuli (oo, $o x, x o$, and $x x$ ), yet some stimuli obviously generate faster RTs than do others. With a crude SOA based on an overall average, it may have been the case, for instance, that a "short" SOA for one participant was short (in regard to occurring before attention had traced too far along the line) only in the $x x$ condition. Likewise, on "long" SOA trials with $x x$ stimuli, the focus of attention may still have been near the top of the stimulus and, therefore, more likely to notice the change. This potential confound was rectified in Experiment 2, with SOAs tailored to each of the four stimulus types for each participant.

\section{EXPERIMENT 2}

Following on from the initial positive results, a second experiment was considered necessary to demonstrate that the effect of reduced accuracy at long SOAs following a change is robust and is not specific to the particular change location used in the first experiment. Accordingly, a second experiment was undertaken that was similar to the first, except for a few notable differences. The primary difference was the location of the change. In Experiment 1, the change always occurred at the top of the stimulus. There is the possibility, however, that something specific to the change location in Experiment 1 produced the results, rather than a moving spotlight of attention. In Experiment 2, the change could occur at either the top or the bottom intersection of the two lines (located at $3^{\circ}$ and $6^{\circ}$ below fixation, respectively). A change would involve either a noncrossing intersection's changing to a crossing intersection or vice versa. For example, an oo trial could change to an $o x$ trial or to an $x o$ trial. As with Experiment 1, any change reversed the response required from the participant. A second modification to the experimental design was to include three SOAs at which the change could occur: short, intermediate, and long. These SOAs were calculated for each stimulus type for each individual on the basis of a baseline block, which was a further improvement over the coarser averages used in Experiment 1. It was predicted that changes that occurred at short SOAs, or at the bottom intersection, might occur before attention reaches the location of the change. In such instances, attention would trace the changed line without the participant's realizing that a change had occurred. At longer SOAs and for changes to the top intersection, it was more likely that changes would occur after attention had passed over the intersection. Such changes should be easily noticed by a spread of attention, but not by a moving zoom lens.

The two modifications to Experiment 2 also allowed a further prediction to be made: If we have chosen our SOAs to correctly reflect tracing at the beginning, middle, and end of the line, the intermediate SOA should discriminate between accuracy rates following changes at the top and at the bottom intersections.

If a change occurs at the short SOA, we assume that a moving zoom lens is near the top of the stimulus above the first intersection. Changes to both the top and the bottom intersections should, therefore, not affect the tracing process, since they occur before attention has reached them. Alternatively, if a change occurs at the long SOA, when we assume that the zoom lens is nearing the bottom of the line, having passed through both intersections, ability to detect the change and reverse the response should be equally poor both for changes that occur at the top intersection and for those that occur at the bottom intersection. However, changes that occur at intermediate SOAs are assumed to correspond to a spotlight that is somewhere in the middle of the stimulus, possibly in between the two intersections. Thus, trials with changes that occur at these intermediate SOAs will receive more correct responses if the change occurs at the bottom intersection, rather than at the top.

\section{Method}

Sixteen new participants were recruited to take part in the study (with a mean age of 25 years; 10 were female). The stimuli and apparatus were identical to those in Experiment 1. A $3 \times 3$ withinsubjects design differed from that in Experiment 1 in three aspects. First, the change could occur at one of two locations, either the top intersection ( $3^{\circ}$ below fixation, accounting for $25 \%$ of all the trials) or the bottom location ( $6^{\circ}$ below fixation; $25 \%$ of all the trials). On the remaining $50 \%$ of the trials, no change occurred. The second change was to introduce a third, intermediate SOA. The final change was in regard to the calculation of the SOAs. Previously, SOAs were based on $10 \%$ and $60 \%$ of the mean RTs to a practice block of 96 tri- 
als without a global flicker or change (minus $200 \mathrm{msec}$ for the motor component of the RTs). For this experiment, $10 \%, 30 \%$, and $60 \%$ of the mean RTs were used (minus $200 \mathrm{msec}$ ) for short, intermediate, and long SOAs, respectively, but these were calculated separately for each of the four stimuli types (oo, $x o, o x$, and $x x$ ). Given that RTs were susceptible to the baseline task in Experiment 1, it seemed prudent to scale all SOAs to stimulus type, to ensure that the assumed location of the spotlight at varying SOAs was as valid for the stimuli that produced the longest tracing times $(x x)$ as it was for the stimuli producing the shortest response times (oo). All other aspects of the methodology were identical to those in Experiment 1.

\section{Results}

As in Experiment 1, the results of the initial practice block that did not contain either a flicker or a change were analyzed first. The overall mean RT was $850 \mathrm{msec}$, with a mean accuracy of $87 \%$, which is comparable to the results of Experiment 1. Both RTs and accuracy measures across the four stimuli types (oo, ox, $x o$, and $x x$ ), were compared in $1 \times 4$ ANOVAs, with repeated planned contrasts comparing $o o$ with $o x$, $o x$ with $x o$, and $x o$ with $x x$.

Main effects of stimulus type were found for both RTs $\left[F(3,45)=30.8, M S_{\mathrm{e}}=7,905.9, p<.001, f=1.43\right]$ and accuracy $\left[F(3,45)=16.0, M S_{\mathrm{e}}=102.4, p<.001, f=\right.$ 1.03]. The RTs were similar to those found in Experiment 1, with $o o$ trials producing the shortest RTs and $x x$ trials producing the longest. However, whereas in Experiment 1 the repeated contrasts revealed differences between $o o$ and $o x$ and between $x o$ and $x x$, the present results showed a difference between $o o$ and $o x\left[F(1,15)=34.6, M S_{\mathrm{e}}=4,780.6\right.$, $p<.001, f=1.52]$ and between $o x$ and $x o[F(1,15)=$ $\left.10.6, M S_{\mathrm{e}}=12,420.7, p<.005, f=0.84\right]$, although the difference between $x o$ and $x x$ failed to reach significance $[F(1,15)=3 \cdot 2]$. Despite some differences in regard to where the significant increases in RTs lie, the overall pattern is very similar to that noted in Experiment 1.

Accuracy scores produced exactly the same pattern of results, with $o o$ and $x o$ trials receiving more accurate responses than did $o x$ trials $\left[F(1,15)=45.3, M S_{\mathrm{e}}=145.6\right.$, $p<.001, f=1.74$, and $F(1,15)=12.6, M S_{\mathrm{e}}=93.7, p<$ $.005, f=0.92$, respectively].

Analysis of the baseline block did not suggest that the participants behaved differently, as compared with the first experiment. Short, intermediate, and long SOAs were calculated from baseline RTs for each stimulus type for each participant. These were used, in the second part of the experiment, to present the global flicker that masked a change on half of the trials. On average, SOAs for the four stimuli types varied from 51 to $77 \mathrm{msec}$ (oo trials to $x x$ trials) for short SOAs, from 154 to $230 \mathrm{msec}$ for intermediate trials, and from 308 to $424 \mathrm{msec}$ for long-SOA trials.

Accuracy scores and RTs were collected from 384 trials across three blocks. Trials were removed from the analyses if a response was made before the flicker (11.6\%). No participants needed to be removed for excessive eye movements. On average, eye movements were made by the participants during the experimental blocks on $1.5 \%$ of all the trials, with the highest number of eye movements for any 1 participant occurring on only $3 \%$ of the trials.
The accuracy scores were subjected to a $3 \times 3$ ANOVA, comparing the change conditions (no change, top intersection change, or bottom intersection change) across the three levels of SOA. A main effect of change was noted $\left[F(2,30)=35.6, M S_{\mathrm{e}}=339.8, p<.001, f=1.54\right]$. Planned contrasts compared the accuracy rates on unchanged trials with the combined accuracy rates for the two change conditions and then compared the two change conditions with each other, assessing accuracy to detect changes at the top intersection, as compared with the bottom intersection. Both contrasts were significant, with the largest effect found in the comparison of the unchanged trials with the changed trials $\left[F(1,15)=45.3, M S_{\mathrm{e}}=203.7, p<.001\right.$, $f=1.73]$. As was expected, accuracy on the unchanged trials was higher than that on the changed trials. A smaller difference was noted for change location, with changes occurring at the bottom intersection producing higher accuracy scores than did changes at the top intersection $\left[F(1,15)=6.0 . M S_{\mathrm{e}}=88.8, p<.05, f=0.63\right]$.

There was also a main effect of SOA $[F(2,30)=27.1$, $\left.M S_{\mathrm{e}}=176.0, p<.001, f=1.34\right]$, with accuracy decreasing with a corresponding increase in SOA.

Most importantly, there was a significant interaction between the two variables $\left[F(4,60)=16.0, M S_{\mathrm{e}}=96.5, p<\right.$ $.001, f=1.03]$. Planned interaction contrasts confirmed that the effects of increasing SOAs on the degradation of accuracy on changed trials was significantly greater than the effects of SOA length on unchanged trials [comparing short with intermediate SOAs across the change/no-change condition produced $F(1,15)=15.7, M S_{\mathrm{e}}=402.3, p<$ $.001, f=1.02$; the same contrast across intermediate and long SOAs gave $F(1,15)=23.5, M S_{\mathrm{e}}=297.4, p<.001$, $f=1.25]$. This interaction can be viewed in Figure $5 \mathrm{~A}$.

The additional hypothesis suggested that intermediate SOAs should best distinguish between accuracy rates following changes in the top intersection and those following changes in the bottom intersection. As can be seen in Figure 5A, the greatest difference between accuracy rates for the top and bottom intersections is found at the intermediate-SOA level, with changes to the bottom intersection faring best, as was predicted. To assess this directly, three planned $t$ tests compared the accuracy on trials containing changes at the two intersections at each of the three SOA levels. Although none of the $t$ tests reached conventional statistical significance, the comparison of top and bottom changes at the intermediate SOA came closest $[t(15)=1.92, p=.075]$.

RTs were again analyzed to identify any possible speedaccuracy trade-off via a $3 \times 3$ within-groups ANOVA. All RTs were recorded from the onset of the original stimulus, as in Experiment 1. The nature of the change was found to be significant $\left[F(2,30)=54.7, M S_{\mathrm{e}}=6,859.0, p<\right.$ $.001, f=1.91]$. RTs to unchanged trials were the shortest (781 $\mathrm{msec})$, followed by responses to changes at the bottom intersection (914 $\mathrm{msec}$ ), with changes to the top intersection producing the longest RTs (949 $\mathrm{msec})$. Planned repeated contrasts confirmed these differences, showing a difference between the unchanged condition and the combined change conditions $\left[F(1,15)=87.5, M S_{\mathrm{e}}=4,114.3, p<.001, f=\right.$ 

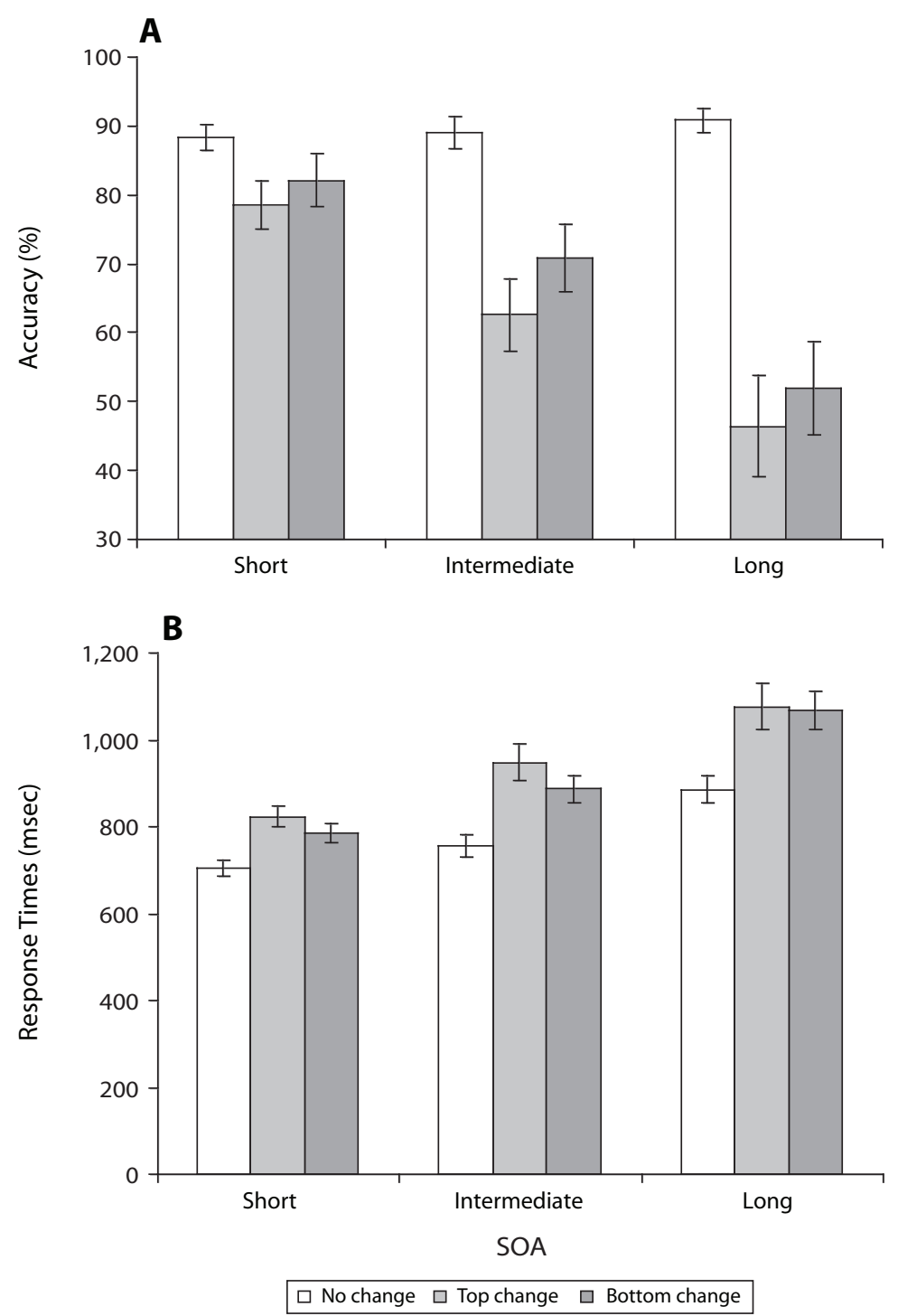

Figure 5. Accuracy of responses (A) and mean response times (B) to the correct target circle for the three change conditions in Experiment 2 (no change, a change at the top intersection, and a change at the bottom intersection) across short, intermediate, and long stimulus onset asynchronies (SOAs). Standard error bars are added.

2.42], and even between the top and the bottom changes $\left[F(1,15)=5.6, M S_{\mathrm{e}}=3,659.4, p<.05, f=0.61\right]$.

A main effect was also noted for SOA $[F(2,30)=54.4$, $\left.M S_{\mathrm{e}}=12,845.4, p<.001, f=1.90\right]$, with planned repeated contrasts confirming that RTs increased from short to intermediate SOAs $\left[F(1,15)=36.6, M S_{\mathrm{e}}=3,757.8\right.$, $p<.001, f=1.56]$ and from intermediate to long SOAs $\left[F(1,15)=45.3, M S_{\mathrm{e}}=7,584.1, p<.001, f=1.74\right]$.

The interaction between these two factors was also significant, although the degrees of freedom were modified by the Greenhouse-Geisser epsilon, due to a deviation from sphericity $\left[F(2.3,34.7)=3.8, M S_{\mathrm{e}}=5,856.8, p<.05, f=0.50\right]$. Although RTs did increase in the unchanged trials with longer SOAs, there was a greater degradation of RTs at longer
SOAs following a change. As can be seen in Figure 5B, this differentiation between changed and unchanged trials occured early in the tracing process, with the interaction being driven by the difference in RTs at the short and intermediate SOAs for the unchanged and changed trials $[F(1,15)=15.2$, $\left.M S_{\mathrm{e}}=4,122.9, p<.001, f=1.01\right]$. As in Experiment 1, these results argue against a speed-accuracy trade-off.

\section{Discussion}

The results of Experiment 2 demonstrate that task accuracy following a change differs according to the location of the change and the moment at which the change occurs during the tracing process. Specifically, changes at the bottom intersection were more likely to be responded to 
correctly, and longer SOAs were associated with higher error rates in the change conditions.

Whereas in the first experiment the change occurred at the very top of the stimulus, thus requiring the new target line to be traced (or at least, a conscious reversal of the response), the second experiment contained changes that could occur before attention had reached the modified intersection. In such trials, the participant may never even have realized that a change had occurred, yet still gave the appropriate response by simply continuing to trace the new pathway of the line.

The spread-of-attention model predicts that participants should be equally accurate on all changes at all SOAs. Some changes will be noticed because attention has already spread through that portion of the line, whereas other changes on parts of the line yet to be traced may not be noticed but will still be responded to correctly. Although these two types of changes may be differentiated by RTs, with the latter producing faster RTs than the former, accuracy should not differ.

The serial zoom lens theory, however, as characterized by Houtkamp et al. (2003), predicts that accuracy will decline with increasing SOAs. The further a participant is into the tracing process when the change is triggered, the more likely it is that the moving spotlight has already passed the intersection before the change occurs. Any changes that occur to portions of the line that have already been traced are, therefore, less likely to be noticed, whereas any changes to untraced portions of the line will be traced as normal, producing the required response.

This theory predicts similar results in regard to RTs: that untraced changes, most likely to the bottom intersection at long SOAs, should be processed quickly without realization that a change has occurred, whereas changes that are spotted within the moving zoom lens produce longer RTs by momentarily delaying the trace or by encouraging participants to retrace the line from fixation.

The decline in accuracy scores suggest that the zoom lens provides a better explanation than does a spreading trace of attention. RTs do not differentiate between the two theories, but it is important to note that there is no evidence of a speed-accuracy trade-off.

An additional prediction was made that intermediate SOAs would be most likely to differentiate between accuracy following changes to the top and bottom intersections. It was suggested that an appropriate SOA could trigger a change while the moving spotlight was in between the top and bottom intersections. In such a case, accuracy for bottom intersection changes should be greater than accuracy for top intersection changes at the intermediate SOA. Although the mean accuracy rates do tend toward this direction, the difference between top and bottom changes narrowly failed to reach conventional statistical significance. Although the pattern is encouraging, the failure to find a clear effect suggests that the intermediate SOA was, perhaps, longer than it needed to be, with the spotlight having already passed the lower intersection on many trials. This does not affect the validity of the main conclusion in favor of the zoom lens, although it does suggest an avenue for future research, further varying the SOAs in an effort to estimate the tracing speed of the zoom lens.

Despite strong evidence from two experiments in favor of a moving spotlight that does not leave an attentional trace, there is, however, a potential confound that could explain the decline in accuracy with longer SOAs, without distinguishing between the two attentional theories. A stimulus-response compatibility problem could arise through the accumulation of information during the tracing process that supports one particular response. When a change occurs that requires a reversal of the response, the difficulty associated with this reversal will be dependent on the strength of the evidence arguing for the prechange response. This evidence is likely to be stronger if the change occurs at long SOAs, since there is a greater likelihood that the participant may have finished tracing the line or, at least, be very close to the end. However, at short SOAs, the participant has less evidence about the prechange target, so that, when a change occurs, the competition between prechange and postchange target evidence is balanced more in favor of the postchange response. If this explanation is valid, the decline in accuracy across increasing SOAs may actually reflect the relative strength of perceptual evidence for the pre- and postchange responses. In a third experiment, we attempted to overcome this problem.

\section{EXPERIMENT 3}

The third experiment required a task that was independent of whether the traced line terminated on the left or the right of the screen. Instead of a response reflecting the terminal location of the target line, the participants were required to respond to a shape at the end of the target line, either a triangle or a square. The rationale for this is that if the target response and the line location are independent, one can withhold target information on the prechange stimulus to prevent competition between prechange and postchange evidence for opposite responses.

To achieve this, each trial began with the same stimuli as those used in both of the previous experiments, with both lines terminating in circles. At varying SOAs, the global flicker was briefly presented before the stimulus reappeared. On half the trials, the flicker masked a change to the lines, with the previous distractor line becoming linked to the fixation circle, as in Experiment 1. In addition, the target circles at the end of both lines changed on every trial to a square and a triangle following the flicker. The participants had to identify which shape was connected to the fixation point.

A further important distinction between this and the previous experiments is that the targets did not appear until after the flicker. Thus, no matter how long the participants may have had to trace the line (short or long SOAs), no prechange response evidence could accrue. Therefore, the competition between pre- and postchange evidence for a particular target response could not occur, and any resultant decrement in accuracy rates for changed stimuli at long SOAs should reflect the lack of attention at an 
earlier point on the line, rather than a systematic stimulusresponse confound.

Furthermore, in the previous experiments, if a stimulus encouraged tracing primarily on the left of the midline, this may have led to further errors if a bottom crossover intersection then required that the participants give a right response. Since the present experiment required responses that were independent of location, this should also avoid any potential Stroop-like interference.

Several other small changes were made to the methodology. Pilot data for Experiment 3 suggested that some participants found it harder to maintain fixation at the top of the stimulus in this task than did the others. In order to more accurately record any errant saccades, the participants' eye movements were recorded with a fixed-bench eyetracker. This allowed us to remove individual trials on which an eye movement was made. Further changes included the removal of feedback from the experimental trials, an emphasis on the importance of the speed of response in participant instructions, and an instruction that told the participants that there might be as many as $50 \%$ of trials that contained a change or as few as $5 \%$. These changes were made in order to reduce the probability of the participants using a strategy in which they might wait until the target shapes appeared after the flicker before tracing the line.

\section{Method}

Participants. Twenty new participants were recruited to participate in Experiment 3 (with a mean age of 20 years; 14 were female). All the participants reported normal or corrected-to-normal eyesight.

Stimuli and Apparatus. The preflicker stimuli were the same as those used in both of the previous experiments. Following the blue flicker, a new stimulus was presented, with a square and a triangle in the place of the two black circles that were the targets in the previous experiments. These new targets were black-filled shapes of a size comparable to that of the previous circles and appeared an equal number of times in each of the two locations.

Pilot studies with these stimuli suggested that the new task was harder than the previous tasks and was more likely to trigger an eye movement away from fixation. In order to more accurately measure the number of trials that produced an errant saccade, the participants' eye movements were monitored with a Hi-Speed eyetracker from SensoMotoric Instruments, which measured the participants' dominant eye at $250 \mathrm{~Hz}$.

The stimuli were presented on a P3 PC with a 21-in. flat-screen monitor. The eyetracker has an integral chinrest that was used to maintain distance from the screen.

Design and Procedure. The design was similar to that in Experiment 1 , with a $2 \times 2$ within-group design consisting of two levels of SOA (short and long), with half of the trials containing a change to the stimulus lines following the flicker. This critical change was the same as that used in Experiment 1, where the prechange distractor line became connected to the fixation point, instead of the prechange target line.

The stimulus that appeared after the flicker had one further change that appeared on all the trials. The two circles that were connected to the ends of the target and distractor line changed into a triangle and a square. Each postflicker stimulus always contained one of each shape, and they were counterbalanced across locations (with an equal number of trials having a square on the right and a triangle on the left and vice versa). Accuracy and RTs were recorded for the participants' decision regarding which of the two shapes was connected to the target line.

The long and short SOAs were again scaled to individual performance (as in Experiments 1 and 2) and were tailored to the four stimulus types (as in Experiment 2). The calculation for the two SOA levels was the same as that used in Experiment 1, except that the proportion of the baseline RT that was removed to account for the motor aspect of the response was doubled to $400 \mathrm{msec}$. This was increased to reflect the more difficult decision-making process. In the previous experiments, the decision was dependent on the tracing process, and the two could not be separated. In the present experiment, however, the independent nature of the task and the tracing process suggested that an additional amount of time would be needed to process the shape once the tracing was completed.

A baseline block of 96 trials preceded the experimental block. These trials did not contain a flicker and had the triangles and squares visible from the start of each trial. As in Experiment 2, RTs for the four stimulus types were used to calculate short and long SOAs for the experimental block.

The instructions were changed slightly to put the emphasis on speed and then accuracy, and feedback was removed from the experimental blocks. The participants were also told that the percentage of trials that could change would be $5 \%, 10 \%, 20 \%, 30 \%, 40 \%$, or $50 \%$, depending on which group they had been randomly allocated to. In reality, all the participants were subjected to $50 \%$ change trials. These changes to the methodology were designed to encourage immediate tracing from stimulus presentation and to discourage a strategy that might favor waiting until after the flicker. This was considered to be especially pertinent for the present experiment, since the target information did not occur until after the flicker. It was also hoped that these changes might decrease the proportion of trials on which thie participants would decide to retrace the line after the flicker, regardless of whether they noticed a change. By limiting these strategies, it was hoped that we could increase the proportion of trials on which the participants missed the change and were duped into giving the wrong answer.

All other aspects of the design and procedure were identical to those in Experiment 1.

\section{Results}

Four participants were removed from all the analyses. One participant scored at chance on the baseline block, and 3 were removed due to either an excessive number of eye movements or calibration problems, resulting in over $75 \%$ of the trials in the experimental block being removed.

As with both of the previous experiments, the RTs and accuracy for the baseline block were analyzed first. The overall mean RTs were 1,125 msec, with a mean accuracy of $75 \%$. The decrease in speed and accuracy, as compared with the baseline block in the first experiment $(879 \mathrm{msec}$ with $82 \%$ accuracy) demonstrates the increased difficulty of the discrimination task. RTs and accuracy rates were compared in separate $1 \times 4$ ANOVAs, with repeated planned contrasts comparing $o o$ with $o x$, $o x$ with $x o$, and $x o$ with $x x$. Main effects of stimulus type were found for both RTs $\left[F(3,45)=7.5, M S_{\mathrm{e}}=14,148, p<.001, f=0.71\right]$ and accuracy $\left[F(3,45)=23.3, M S_{\mathrm{e}}=104.6, p<.001, f=1.25\right]$.

The RTs followed a pattern similar to that in the previous experiments, with more complex stimuli taking longer to trace, although the location of the significant differences was slightly different. Longer RTs were noted for $x x$ stimuli than for $x o$ stimuli $\left[F(1,15)=4.45, M S_{\mathrm{e}}=\right.$ $38,244, p=.05, f=0.54]$ and for $x o$ trials than for $o x$ trials $\left[F(1,15)=7.6, M S_{\mathrm{e}}=14,397, p<.05, f=0.71\right]$. However, oo stimuli did not produce faster RTs than did ox stimuli, as they had in both of the previous experiments $[F(1,15)=1.7]$.

Accuracy scores produced a similar pattern, with a large drop off in accuracy for $x x$ trials, as compared with $x o$ tri- 
als $\left[F(1,15)=41.0, M S_{\mathrm{e}}=157.4, p<.001, f=1.65\right]$, and no difference between $o o$ and $o x$ stimuli.

Although the results fit the general pattern noted in the previous two experiments, with accuracy decreasing and RTs increasing with more complex stimuli, the failure to find any differences between $o o$ and $o x$ trials suggests that the increased difficulty of the shape discrimination task swamped any advantage given by the fastest tracing process with the oo stimuli. This difference between the present baseline results and those in the two previous experiments is not crucial to the main hypothesis, however. These RT results for each stimulus type formed the basis of the short and long SOAs used in the main experimental block.

Before analyzing the experimental block, all trials on which an eye movement was made, or on which calibration was momentarily lost, were removed from the calculation of accuracy rates and RTs. Eye movements that shifted the point of gaze more than $2^{\circ}$ from the fixation point were considered to be serious enough to remove, since they potentially placed the new point of gaze within $1^{\circ}$ of the top intersection. Across all 16 remaining participants, a total of $22 \%$ of the trials were removed, due to eye movements or calibration problems.

The accuracy scores were subjected to a $2 \times 2$ ANOVA, comparing the presence or absence of a change across short and long SOAs. A main effect of change was noted $\left[F(1,15)=19.5, M S_{\mathrm{e}}=23.4, p<.001, f=1.14\right]$, with accuracy on the unchanged trials higher than that on the changed trials ( $85 \%$ and $80 \%$, respectively). There was also a main effect of SOA $\left[F(1,15)=11.4, M S_{\mathrm{e}}=23.0\right.$, $p<.005, f=0.87$ ], showing a significant decrease in accuracy with a corresponding increase in SOA. The interaction between these two factors was also significant $\left[F(1,15)=19.0, M S_{\mathrm{e}}=16.6, p<.001, f=1.13\right]$. As can be seen in Figure 6A, this interaction was due to the participants' reduced ability to give the correct response when a change occured at a long SOA.

Response times were again analyzed to identify any possible speed-accuracy trade off via a $2 \times 2$ withingroup ANOVA. All RTs were recorded from the onset of the original stimulus, as in Experiment 1.

The nature of the change was found to be significant $\left[F(1,15)=209, M S_{\mathrm{e}}=1279, p<.001, f=3.73\right]$, with unchanged trials $(1,163 \mathrm{msec})$ producing faster RTs that did changed trials $(1,293 \mathrm{msec})$. The magnitude of the difference between changed and unchanged trials was similar to that noted in both of the previous experiments. A main effect was also noted for SOA $\left[F(1,15)=434.6, M S_{\mathrm{e}}=\right.$ $3,727.6, p<.001, f=5.38$ ], with faster RTs on trials with short SOAs. The interaction between these two factors was also significant $\left[F(1,15)=16.3, M S_{\mathrm{e}}=1,020.9\right.$, $p<.001, f=1.04]$. Figure $6 \mathrm{~B}$ demonstrates that although a detected change to the stimulus will slow RTs following a short SOA, detection of changes at a long SOA leads to a greater degradation of RTs. These results do not suggest that the accuracy results can be explained in terms of a speed-accuracy trade-off.

Throughout all the experiments, the type of stimulus (oo, ox, xo, and $x x$ ) has been included only in the analysis of the baseline data, which was important in order to
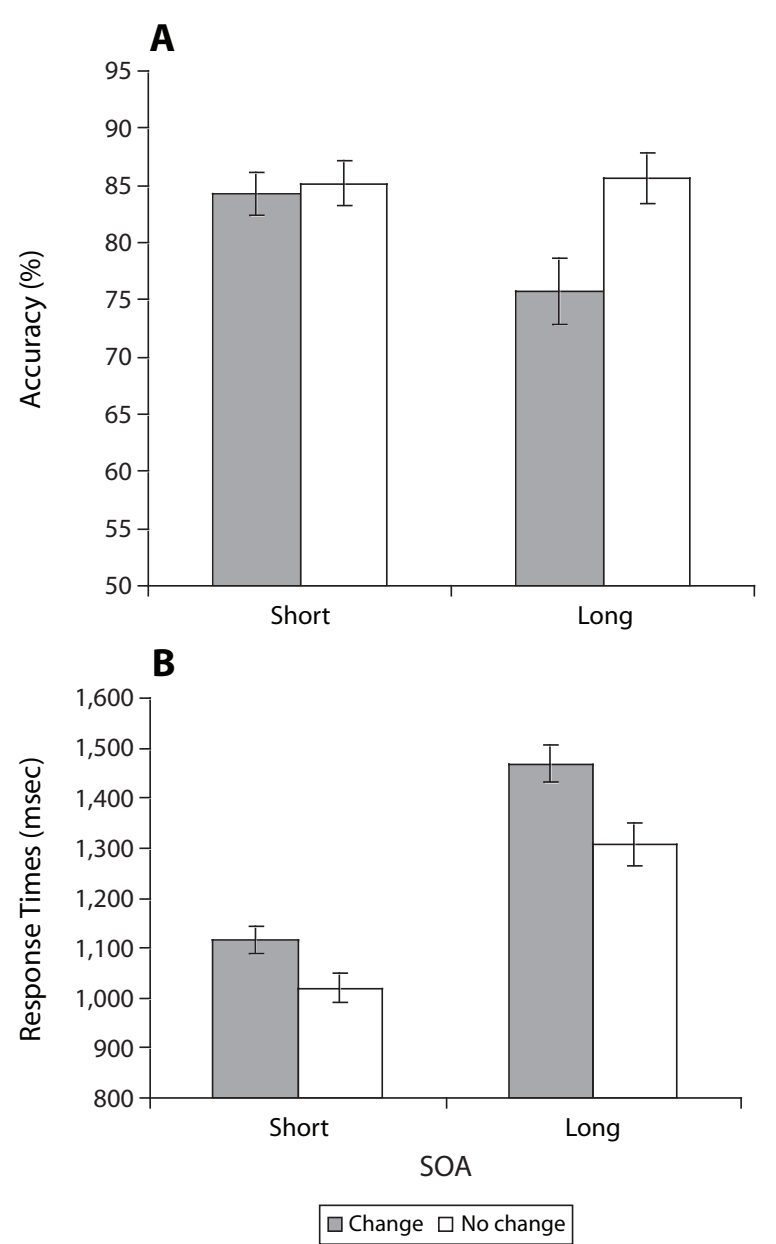

Figure 6.Accuracy of responses (A) and mean response times (B) in Experiment 3 for the correct target shape according to whether a change was present or absent, across short and long stimulus onset asynchronies (SOAs). Standard error bars are added.

confirm that the line-tracing task produces a basic performance similar to that in previous studies (e.g., Houtkamp et al., 2003) and to calculate SOAs specific to stimulus type (Experiments 2 and 3). Stimulus type was not included as a factor in the main analyses, since it was not considered important to the main hypothesis, which predicted the interaction between the presence or absence of a change and the length of the SOA. In order to assess whether the stimulus type had any influence upon the results for this final experiment, however, a series of post hoc $t$ tests were conducted comparing the accuracy scores of participants on changed and unchanged trials across the four stimulus types for both short and long SOAs. As can be seen in Figure 7, although there were overall differences between the four stimuli types (with $x x$ trials producing the lowest levels of accuracy), responses to changed trials with long SOAs across all the stimuli were consistently worse than those in the unchanged trials. With short-SOA trials, however, there was only one stimulus type that showed a slight effect favoring the unchanged 
trials, although on the whole, there was little degradation of the accuracy of responses across all stimuli at short SOAs. These results suggest that the decreased accuracy at long SOAs is not caused by one particular stimulus but is evident in them all.

\section{GENERAL DISCUSSION}

Three experiments consistently demonstrated impairment to response accuracy if a change to the stimulus occured at a relatively long SOA, which then required a reversal to the task response. Changes that occurred at short SOAs were detected more readily, however, and had less of an impact upon accuracy ratings. This is predicted by Houtkamp et al.'s (2003) description of the zoom lens model but cannot be accounted for by the spreading trace hypothesis, which suggests that attention should be present in equal measure at all parts of a line that have already been traced.

We have also demonstrated that this effect is not an aberration caused by the location of the change. Across the three experiments, changes occurred to the top of the stimuli and at each of the intersections. Degradation of accuracy followed predictions of the serial zoom lens hypothesis in every case.

Neither does the effect appear to depend on the complexity of the stimuli. All four stimulus types have shown a significant degradation from unchanged to changed trials at long SOAs. Furthermore, the third experiment addressed issues of stimulus-response compatibility, which may have confounded the results. Even when these potential confounds were controlled for, the results clearly demonstrated a larger degradation in response accuracy at long SOAs than at short SOAs.

It was noticeable in the accuracy data that although a significant degradation was noted for long-SOA trials following a change, the percentage of correct responses was still considerably high. One might argue that accuracy rates of $60 \%-75 \%$ in the changed trials at long
SOAs could not be achieved without some attentional resources being left in the wake of the moving zoom lens. This could indeed be the case. McCormick and Jolicour (1994) never suggested that the zoom lens did not leave an attentional trace or marker on the segments of the line that had already been passed over, and their argument that line tracing forms a part of figure-ground separation (McCormick \& Jolicœur, 1992) indeed implies that some form of marker must be left behind. The clear distinction between a spreading trace and a zoom lens that leaves no trace was made only by Scholte et al. (2001) and Houtkamp et al. (2003). The only distinction of importance for the present authors is that the spreading trace hypothesis suggests that attention must be allocated to all parts of the traced line in equal measure. The results of the present study argue that this is not the case and that, if attentional resources are left on the traced portions of the line after the zoom lens has passed over, these resources are attenuated, as compared with the resources in the moving focus of the zoom lens. An alternative explanation for the relative high accuracy rates is that the chosen SOAs were too short, perhaps resulting in a percentage of trials on which even at a "long" SOA, the zoom lens was still near enough to the top of the stimulus to spot any changes. For this reason, acknowledged in the discussion of Experiment 1, it is safer to discuss relative changes in accuracy rates, rather than absolute changes. In terms of relative accuracy, the results definitely argue against Houtkamp et al.'s spreading attention model.

In regard to the RT data, analyses confirmed that the accuracy results were not due to a speed-accuracy trade-off. Correct responses in the crucial change condition at long SOAs took considerably longer than in comparable conditions across all the experiments. This RT delay on trials following a change is likely to be due to the participant's thinking that a change may have occurred and then retracing the line to make sure (either due to the proximity of the zoom lens to the change locus or through attenuated re-

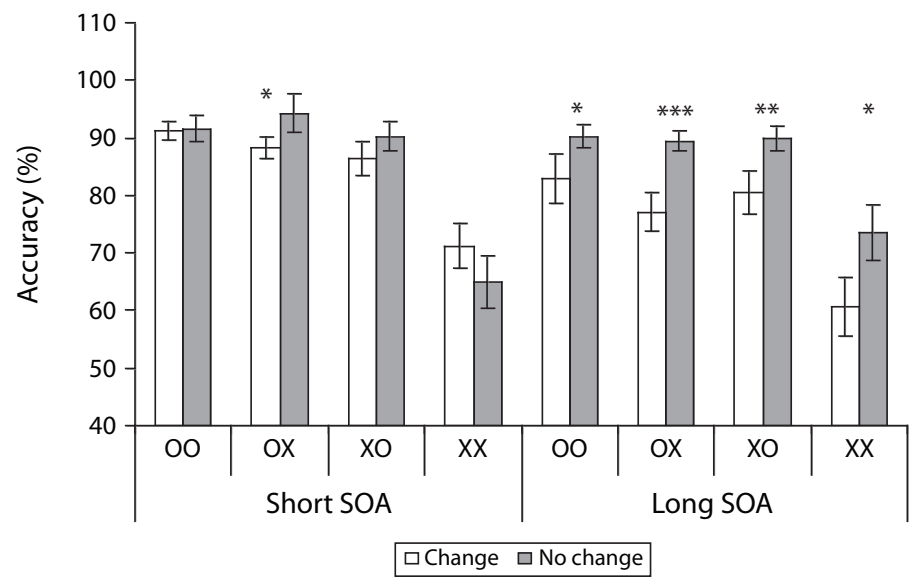

Figure 7. Accuracy rates for Experiment 3 broken down across the four stimuli types. Standard error bars are added. "Difference between the changed and the unchanged trials significant at $p<.05$, using post hoc $t$ tests. ${ }^{* *}$ Difference significant at $p<.01$. ${ }^{* * *}$ Difference significant at $p<.005$. 
sources left on the traced portions of the line). This effect may be pronounced for changes at long SOAs because of a decrease in the signal-to-noise ratio regarding the detection of the change. The less certain the participant is about the occurrence of a change (because attention has moved further from the change locus at long SOAs), the longer it takes before a decision is made to reorient attention to that change (Bundesen, 1990).

In addition to the degrading effect of a change on RTs, the longer SOAs slowed RTs, regardless of the presence of a change. It is possible that the farther covert attention moves from the point of fixation into the extrafoveal region, the more difficult the tracing task may become, due to the decreasing resolution of the retina. A flicker at a long SOA may, therefore, be more likely to confuse the line-tracing operator, or at least make the participant doubt that he or she is still tracing the correct line (regardless of whether a change was perceived). Thus, flickers at long SOAs may increase the number of trials on which the participant retraces the line.

To summarize the RT data, rather than providing any evidence in favor of a speed-accuracy trade-off, they appear to support the hypothesis that tracing a line requires a serial focus of attention, as is demonstrated by the accuracy results.

The overall conclusion, which argues against Houtkamp et al.'s (2003) spreading trace model, hinges on two assumptions. The first assumption is that line tracing did indeed occur. It could, instead, be argued that attention merely needs to jump from one intersection to another to complete the task. Scholte et al. (2001) and Houtkamp et al. have, however, produced evidence against this alternative strategy. Although we have questioned the use of their secondary task to distinguish between a spread of attention or the movement of attention (reporting the colors of the arches), we have no concerns over the evidence they present to demonstrate that line tracing does occur with these stimuli. Furthermore, the line-tracing strategy is functionally simpler than an intersection strategy, which requires the participant to remember the start state of the line (whether it emerged from the left or the right of fixation) and then modify this according to the state of the two following intersections. Tracing a line is a single process that does not require such complex cognitive operations. Certainly, none of our debriefed participants reported using this strategy.

The second assumption upon which the current conclusions lie is that if attention was devoted equally to all of the traced line at the point at which the change occurred, the change should be detected easily, regardless of where in the line the change appears. One potential problem with this assumption, however, is that although attention to an object may be necessary to detect a change in that object, there is a question over whether it is sufficient. Although some researchers have argued that devoting attention to an object will result in the processing of all the features of that object, including task-irrelevant features (Duncan, 1993; O'Craven, Downing, \& Kanwisher, 1999), there is, however, evidence to suggest that some task-irrelevant features may not be processed in all cases. Scholl, Pylyshyn, and Franconeri (1999) asked participants to track multiple dynamic objects moving among distractors. Masked changes would occur on occasion to either a distractor or one of the targets. When the change affected a target's color or shape, there was no improvement for change detection in targets, as compared with distractors. However, when the change affected a target's location or heading (both features that were more directly related to the tracking task than was shape or color), change detection was found to be superior in targets.

In the present tasks, the location of the target line relative to the distractor line was vital to the task, even in Experiment 3 , in which the ultimate decision was based on the shape of the target. Thus, even though we may suggest that attention to the target line might not process all the features of that line (color, thickness, etc.), its location and relationship with both the fixation point and the distractor line are relevant features that must be processed in order to complete the task. If we had used a color change that appeared at the top portion of the target line, the possibility that only task-relevant features of the object were processed could have confounded the results. Since the change affected a task-relevant feature, the effect reported by Scholl et al. (1999) should not pose a problem for the present results.

With such strong evidence against the spreading trace hypothesis, we must consider what ramifications these results have for the incremental-grouping process (Roelfsema et al., 2000; Scholte et al., 2001). The fact that some objectparsing processes require attention has been previously noted (e.g., Ben-Av, Sagi, \& Braun, 1992), and this implies that there is some form of effortful process for grouping certain features. If one accepts that incremental grouping is a likely process for effortful grouping of unusual features, we are left with two strong alternative conclusions. Either incremental grouping does not require a spread of attention through a collection of features, or the process of line tracing does not involve incremental grouping (and therefore, does not represent an instance of object parsing).

In regard to the first alternative, one could suggest that incremental grouping may be completed by a moving focus of attention. For instance, the moving zoom lens may build up a memory of the object as it traces it, cumulatively adding to an object file until the tracing process is completed and the object is considered parsed. This would not require attention to be at all points on a line concurrently and could lead to an incorrect object perception if an undetected change occurs that alters the object before the incremental-grouping process has been completed. Alternatively, as was mentioned above, an attenuated attentional trace may be left on those portions of a line that have already been traced. These resources would not be as great as the level of attention devoted to the serial zoom lens but still might be strong enough to flag up a change to the traced line in some circumstances (possibly explaining why participants can still achieve a $60 \%$ accuracy rate following a change in the long-SOA condition). Other possibilities could include a moving spotlight as a precursor to a spread of attention: If the moving spotlight reaches the end of a particular stimulus (or has returned to the point of fixation after tracing the outline of a closed figure), the features might then be considered eligible for grouping. 
Attention would then spread through the features as the object representation is created. Although various conceptions of incremental grouping with a moving zoom lens could be posited, such speculation goes beyond the present results, especially since it is not clear whether a traced line is even perceived as an object.

This brings us to the second alternative: that line tracing does not involve incremental grouping and does not produce an object representation. A moving attentional focus is quite sufficient to complete the task, and in previous line-tracing tasks (e.g., Jolicœur et al., 1986), there has been no reason to create an object representation, since once the target was reached, the lines disappeared and were replaced by a new stimulus. One could argue, however, that the present study does provide an incentive to see the line as a whole object once tracing has been completed, for this would allow the participants to check whether a change had occurred without reorienting a focus of attention back to the top of the line. If this had happened, accuracy for trials with a change at long SOAs should have still remained as high as in short-SOA trials. Evidence against line tracing's producing object representations has also been found by Wolfe, Klempen, and Dahlen (2000). They attempted to produce robust representations of traced lines by presenting participants the same lines for 100 trials at a time. The only thing that changed was the location of the two target stimuli (in regard to separation along the same line or appearing on different lines). The two lines remained on the screen throughout the block. Even over this large number of trials, Wolfe et al. found no evidence that tracing rates for targets on the same line improved, suggesting that the participants did not build up representations of the lines over time.

Of the two alternatives, it is prudent to assume that until line-tracing studies have produced evidence to show that a traced line is subsequently represented as an object, incremental grouping does not occur during the serial movement of the focus of attention along the line. Instead, line tracing may simply be a specific strategy employing a moving focus of attention to deal with stimuli that do not occur on a regular basis and for which there is either no advantage to or no mechanism for storing them as a single object representation.

\section{AUTHOR NOTE}

The authors thank Jane Arthur and Rebecca Curling for assistance with data collection and Peter McCormick, Brad Gibson, and Steven Sholte for their insightful comments on earlier versions of the manuscript. Correspondence concerning this article should be addressed to D. Crundall, School of Psychology, University of Nottingham, University Park, Nottingham NG7 2RD, England (e-mail: david.crundall@ nottingham.ac.uk).

\section{REFERENCES}

Ben-Av, M. B., SAGI, D., \& Braun, J. (1992). Visual attention and perceptual grouping. Perception \& Psychophysics, 52, 277-294.

Bundesen, C. (1990). A theory of visual attention. Psychological Review, 97, 523-547.
Cole, G. G., Kentridge, R. W., \& Heywood, C. A. (2005). Object onset and parvocellular guidance of attentional allocation. Psychological Science, 16, 270-274.

DunCan, J. (1993). Similarity between concurrent visual discriminations: Dimensions and objects. Perception \& Psychophysics, 54, 425430.

Hazlett, C. J., \& Woldorff, M. G. (2004). Mechanisms of moving the mind's eye: Planning and execution of spatial shifts of attention. Journal of Cognitive Neuroscience, 16, 742-750.

Houtkamp, R., Spekreise, H., \& Roelfsema, P. R. (2003). A gradual spread of attention during mental curve tracing. Perception \& Psychophysics, 65, 1136-1144.

JolicEUR, P., \& IngLETON, M. (1991). Size invariance in curve tracing. Memory \& Cognition, 19, 21-36.

Joliceur, P., Ullman, S., \& Mackay, M. (1986). Curve tracing: A possible basic operation in the perception of spatial relations. Memory \& Cognition, 14, 129-140.

Joliceur, P., Ullman, S., \& Mackay, M. (1991). Visual curve tracing properties. Journal of Experimental Psychology: Human Perception \& Performance, 17, 997-1022.

$\mathrm{Lu}, \mathrm{S}$. (2006). Cue duration and parvocellular guidance of visual attention. Psychological Science, 17, 101-102.

McCormick, P. A., \& Joliceeur, P. (1991). Predicting the shape of distance functions in curve tracing: Evidence for a zoom lens operator. Memory \& Cognition, 19, 469-486.

McCormick, P. A., \& Joliceur, P. (1992). Capturing visual attention and the curve tracing operation. Journal of Experimental Psychology: Human Perception \& Performance, 18, 72-89.

McCormick, P. A., \& Joliceeur, P. (1994). Manipulating the effects of distance effects in visual curve tracing: Further evidence for the zoom lens model. Canadian Journal of Experimental Psychology, 48, 1-24,

O’Craven, K. M., Downing, P. E., \& Kanwisher, N. (1999). fMRI evidence for objects as the units of attentional selection. Nature, 401, 584-587.

Rensink, R. A. (2000). The dynamic representation of scenes. Visual Cognition, 7, 17-42.

Rensink, R. A., O'Regan, J. K., \& Clark, J. J. (1997). To see or not to see: The need for attention to perceive changes in scenes. Psychological Science, 8, 368-373.

Roelfsema, P. R., Lamme, V. A. F., \& Spekreijse, H. (2000). The implementation of visual routines. Vision Research, 40, 1385-1411.

Scholl, B. J., Pylyshyn, Z. W., \& Franconeri, S. L. (1999). When are featural and spatiotemporal properties encoded as a result of attentional allocation? [Abstract]. Investigative Ophthalmology \& Visual Science, 40, 797.

Scholte, H. S., Spekreijse, H., \& Roelfsema, P. R. (2001). The spatial profile of visual attention in mental curve tracing. Vision Research, 41, 2569-2580.

Simons, D. J. (1996). In sight, out of mind: When object representations fail. Psychological Science, 7, 301-305.

Simons, D. J. (2000). Current approaches to change blindness. Visual Cognition, 7, 1-16.

Sumner, P., Adamjee, T., \& Mollon, J. D. (2002). Signals invisible to the collicular and magnocellular pathways can capture visual attention. Current Biology, 12, 1312-1316.

TheEuwes, J. (1995). Abrupt luminance change pops out; abrupt color change does not. Perception \& Psychophysics, 57, 637-644.

Wolfe, J. M., Klempen, N., \& Dahlen, K. (2000). Postattentive vision. Journal of Experimental Psychology: Human Perception \& Performance, 26, 693-716.

Wong, E., \& Weisstein, N. (1983). Sharp targets are detected better against a figure, and blurred targets are detected better against a background. Journal of Experimental Psychology: Human Perception \& Performance, 9, 194-202.

(Manuscript received February 2, 2005; revision accepted for publication September 12, 2007.) 